IdeAs

Idées d'Amériques

$10 \mid 2017$

États-Unis / Cuba : une nouvelle donne?

\title{
Jacques Galinier et Aurore Monod Becquelin, Las cosas de la noche, una mirada diferente
}

Mexico, Edition CEMCA, 2016, 241 p.

Delphine Mercier

\section{(2) OpenEdition}

\section{Journals}

Édition électronique

URL : https://journals.openedition.org/ideas/2175

DOI : $10.4000 /$ ideas. 2175

ISSN : 1950-5701

Éditeur

Institut des Amériques

Référence électronique

Delphine Mercier, « Jacques Galinier et Aurore Monod Becquelin, Las cosas de la noche, una mirada diferente », IdeAs [En ligne], 10 | 2017, mis en ligne le 18 décembre 2017, consulté le 19 octobre 2022. URL : http://journals.openedition.org/ideas/2175 ; DOI : https://doi.org/10.4000/ideas.2175

Ce document a été généré automatiquement le 19 octobre 2022.

\section{c) (i) $\ominus$}

Creative Commons - Attribution - Pas d'Utilisation Commerciale - Pas de Modification 4.0 International - CC BY-NC-ND 4.0

https://creativecommons.org/licenses/by-nc-nd/4.0/ 


\title{
Jacques Galinier et Aurore Monod Becquelin, Las cosas de la noche, una mirada diferente
}

Mexico, Edition CEMCA, 2016, 241 p.

\author{
Delphine Mercier
}

\section{RÉFÉRENCE}

Jacques Galinier et Aurore Monod Becquelin, Las cosas de la noche, una mirada diferente, Mexico, Edition CEMCA, 2016, 241 p.

1 Dès sa constitution, le groupe de recherche «Anthropologie de la nuit » (Laboratoire d'ethnologie et de sociologie comparative (CNRS-Université Paris Ouest) s'est donné pour objectif de réunir des spécialistes de plusieurs disciplines telles que la sociologie, l'histoire, l'ethnolinguistique, le droit et l'économie, pour aborder les pratiques quotidiennes ou rituelles propres à la nuit, dans leurs contraintes et leurs aménagements, en considérant aussi bien l'analyse des systèmes de croyances éclairés par chacune des disciplines mentionnées que par l'écologie, ou la philosophie. Ce domaine, qui recouvre une part considérable des activités humaines, conscientes et inconscientes, concerne l'ensemble des objets et des processus étudiés par l'anthropologie, dans toutes ses directions, c'est à dire aussi bien le corps et ses techniques, la notion de personne, que la culture matérielle, les échanges, et les représentations. La multiplicité des points de vue sur la nuit appelle le concours des disciplines dont la collaboration est d'autant plus nécessaire que la recherche présente un nombre considérable de lacunes tant théoriques que méthodologiques. Les principales absences ethnographiques et théoriques, telles qu'elles ressortent de la 
description des faits sociaux considérés dans leur spécificité nocturne peuvent être ainsi brièvement énumérées :

- peu de faits systématiquement récoltés en vue d'une étude de la nuit, et moins encore de visée comparative ;

- une dépendance méthodologique et descriptive de la nuit, conçue presqu'exclusivement comme complémentaire ou inverse du jour, alors que la «nocturnité » implique une idée de transformation, induite par les changements qui affectent les données physiques extérieures et internes au corps- expérimentées par les humains, et l'interprétation culturelle qu'ils en fournissent ;

- une absence de réflexion générale sur l'étendue et les limites des particularités qu'introduisent les humains dans leur conception de la nuit.

2 Après avoir parcouru le territoire de la nocturnité depuis de nombreux points de vue et dans des terrains fort divers, le groupe "Anthropologie de la Nuit» a conclu à la nécessité de proposer une nouvelle approche de ce domaine, dont le colloque de Mexico organisé en 2012 devrait présenter les axes majeurs. Cette démarche implique de repenser le programme de l'enquête ethnographique (étude de la morphologie sociale, de l'action), sur la base de terrains proprement «nocturnes ", pour remonter vers les ordres sociaux « diurnes », décrits par les chercheurs et dans les théories indigènes. Les observations de terrain sont axées sur les processus de construction de ces ordres de la nuit, leur structure, leur fonctionnement, leur économie et leurs normes juridiques, leur dynamique et leurs désajustements. Il s'agit de comprendre les règles imposées par la nuit, celles que certains types d'action imposent à la nuit et enfin les règles que la nuit impose au jour, à savoir comment les pratiques nocturnes affectent, souvent considérablement, celles du jour. Des recherches précises et concentrées ont été poursuivies en gardant la multiplicité et la variété des terrains : les contraintes - les veilles, les états liminaires ou altérés de la conscience comme les visions, et même les fardeaux de la nuit (non seulement les dépenses énergétiques, économiques, symboliques mais les dépenses en émotions, transgressions, obligations (de raconter les rêves pour contrer les mauvais présages et régler la vie diurne).

Cette approche implique également de repenser la communication nocturne. Ce champ de l'interaction multimodale, est conventionnellement jugé comme "altéré » si on le conçoit comme en contraste avec le jour. En revanche, si l'on en détaille les propriétés spécifiques qui incluent le sommeil, l'activité onirique, les conceptions du corps, les perceptions relatives au monde, il devient à la fois autonome et interdépendant avec le monde diurne. Cette publication marque donc une nouvelle étape dans un projet mis en lumière dans le cadre du séminaire «Anthropologie de la nuit » et s'inscrit dans un courant de réflexion qui trouve aujourd'hui une croissance constante dans le monde et qui vise à appréhender ce nouvel objet d'une perspective transnationale et interdisciplinaire. Cet ouvrage, issu d'un projet de recherche portant sur les définitions et les limites de la nuit dans différentes cultures à des fins de comparaison, soumet à une analyse critique le lieu commun qui associe constamment la nuit à l'obscurité et permet de construire des méthodes innovantes, ainsi que des outils analytiques spécifiques. Les textes rassemblés dans cet ouvrage correspondent aux actes du Colloque Las Cosas de la Noche tenu à Mexico les 18 et 19 octobre 2012.

4 La nuit est certainement l'un des sujets les plus prometteurs de l'anthropologie du nouveau millénaire sur lequel est implanté maintenant une foule de séminaires, thèses et domaine de la recherche. Si la nuit était restée dans l'ombre dans les analyses 
anthropologiques c'est essentiellement car le regard, les habitudes et l'expérience de l'Occident sur l'autre moitié de notre existence semblaient rationnels et universels, alors que ceux des autres, l'espace et le temps exotique, non seulement semblaient surprenants et incompréhensibles, mais aussi follement irrationnels. En fait, nous avons l'habitude de penser et de vivre un certain nombre d'alternances qui paraissent évidentes ou naturelles: clarté de jour/obscurité nocturne, sillage/sommeil, activité / repos, sécurité / danger. Il est difficile pour nous de nous convaincre que d'autres sociétés ne les conçoivent pas et ne les vivent pas de la même façon. Les essais présentés dans cet ouvrage suggèrent ces splendeurs de la vie nocturne, de mondes préhispaniques à nos espaces urbains contemporains, de Papouasie à la mafia italienne, chamans amazoniens dans l'Arctique immergé dans la longue nuit polaire de six mois. Enfin, il apparait que les anthropologues de la nuit tout comme les artistes, les musiciens et les poètes « croient à la nuit », Rainer Maria Rilke

\section{NOTES}

1. Rainer Maria Rilke, Poémes à la nuit, Der Doppelgänger, Édition bilingue. Traduit de l'allemand et présenté par Gabrielle Althen et Jean-Yves Masson. Préface de Marguerite Yourcenar, 112 p., janvier 1994.

\section{AUTEURS}

\section{DELPHINE MERCIER}

Aix Marseille Université, LEST, Aix-en-Provence 